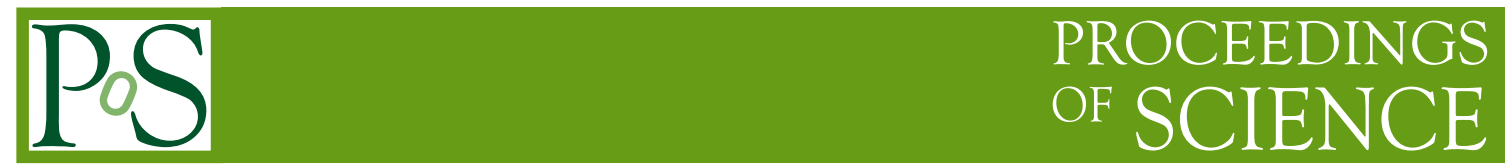

\title{
Status of $\mathrm{JSNS}^{2}$ experiment
}

\author{
F. Suekane*t \\ RCNS, Tohoku Univ. Japan \\ E-mail: suekanedawa.tohoku.ac.ip
}

JSNS $^{2}$ is an experiment which aims to search for a sterile neutrino by $\bar{v}_{\mu} \rightarrow \bar{v}_{e}$ appearance mode using $\bar{v}_{\mu}$ from muon decay at rest. This method is the same as the LSND experiment and JSNS ${ }^{2}$ will perform direct test of the LSND positive result with much better performances. In addition, JSNS $^{2}$ will perform experiment using monoenergetic $v_{\mu}$ from $\mathrm{K}^{+}$decay at rest (KDAR). These experiments are being prepared at the J-PARC MLF and are planned to start data taking from early 2020 .

The 21st international workshop on neutrinos from accelerators (NuFact2019)

August 26 - August 31, 2019

Daegu, Korea

${ }^{*}$ Speaker.
${ }^{\dagger}$ For JSNS ${ }^{2}$ collaboration

(C) Copyright owned by the author(s) under the terms of the Creative Commons 


\section{Introduction}

In the standard model, the number of neutrino flavors is three. The number comes from the observation that the mass width of the weak neutral gauge boson $Z^{0}$ is consistent with just 3. If there would be 4 th neutrino $\left(v_{4}\right)$, the lifetime of the $Z^{0}$ would be shorter due to the additional decay channel $\left(Z^{0} \rightarrow v_{4} \bar{v}_{4}\right)$ and the mass width would be wider than the observation. However, this observation does not rule out existence of neutrinos which do not perform weak interactions, or neutrinos whose masses are heavier than half of the $Z^{0}$ mass. There have been several experimental observations which can not be explained by the standard three neutrinos and imply existence of additional neutrinos with $\mathrm{eV}$ mass scale [四]. To live with the $Z^{0}$ mass width, those neutrinos are assumed not to perform the weak interactions. We call those weakly non-interacting neutrinos, as sterile neutrinos.

LSND experiment is a pioneering experiment which showed indications of sterile neutrinos [0]. LSND group observed $\bar{v}_{\mu} \rightarrow \bar{v}_{e}$ oscillation at $\Delta m^{2}>0.03 \mathrm{eV}^{2}$, which can not be accounted by the standard three neutrinos, with significance $3.8 \sigma$. This had been the most significant indication of sterile neutrino for long time until recently. In the LSND experiment, $\bar{v}_{\mu}$ was produced in the decay of $\mu^{+}$stopped in the target material. The $\bar{v}_{e}$ was detected by using the inverse beta decay reaction $\left(\bar{v}_{e}+p \rightarrow e^{+}+n\right)$ in a liquid scintillator.

JSNS $^{2}$ (J-PARC Sterile Neutrino Search at J-PARC Spallation Neutron Source) group was formed aiming at testing the LSND positive result using the same decay at rest $\bar{v}_{\mu}$, the same $\bar{v}_{e}$ detection methods, similar baseline, yet much better signal-to-noise ratio, energy resolution and statistics. In addition, thanks to the high beam energy $(3 \mathrm{GeV})$, JSNS ${ }^{2}$ can make use of monoenergetic $v_{\mu}(236 \mathrm{MeV})$ from $\mathrm{K}^{+}$decay at rest. This neutrino was first observed by MiniBooNE experiment [[] and JSNS ${ }^{2}$ can perform clean experiments using this unique neutrino.

\section{JSNS ${ }^{2}$ experiment}

Fig. $\square$ shows JSNS ${ }^{2}$ neutrino detector (the 1 st $^{\text {phase }}{ }^{1}$ ) and J-PARC MLF beam line. The neutrino detector will be placed on the 3rd floor of the J-PARC MLF building. A 3GeV 1MW(planned) proton beam is sent to the mercury target of the neutron spallation source at the end of the MLF beam line. About $0.3 \bar{v}_{\mu}$ per incident proton are produced in the decay of $\mu^{+}$at rest in the mercury target. Since the $\mu^{+}$decays after it stops, the energy spectrum of $\bar{v}_{\mu}$ is determined by the electroweak theory without ambiguity. The produced neutrinos spread out isotropically. Therefore, there is no geometrical uncertainty of the neutrino flux at the detector.

A big advantage of JSNS ${ }^{2}$ over the LSND is pulsed beam. The MLF proton beam consists of two $80 \mathrm{~ns}$ short pulses separated by $540 \mathrm{~ns}$ apart. This double pulses come every $40 \mathrm{~ms}(25 \mathrm{~Hz})$. The lifetime of $\mu^{+}$is $2.2 \mu \mathrm{s}$ and by setting the $\bar{v}_{\mu}$ timing window as $1 \sim 10 \mu \mathrm{s}$, the accidental backgrounds are suppressed to $2 \times 10^{-4}$ without loosing $\bar{v}_{\mu}$ so much. The prompt beam backgrounds can also be removed significantly[[]].

Fig. $\square$ shows the neutrino detector. It consists of, from inner to outer, 17tons of Gadolinium loaded liquid scintillator (neutrino target) contained in a cylindrical acrylic vessel. 34 tons of un-loaded liquid scintillator which is optically separated into $\gamma$-catcher (inner) and anti-counter

\footnotetext{
${ }^{1}$ The 2 nd phase would consist of two detectors of different baselines.
} 


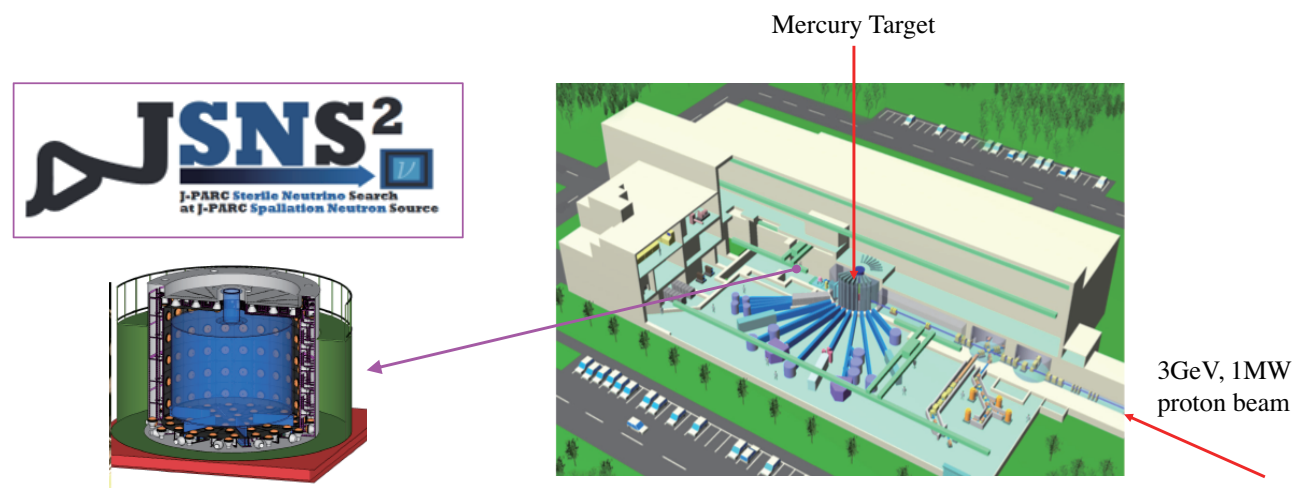

(a)

(b)

Figure 1: (a) JSNS ${ }^{2}$ detector will be placed at the 3rd floor of the building and the beam line. $(b)$ J-PARC MLF building.

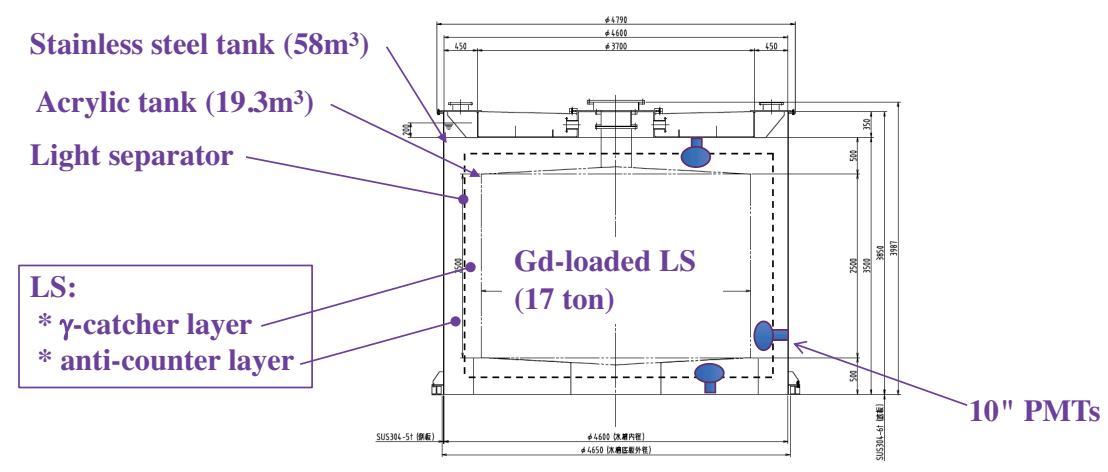

Figure 2: Cross section of the $\mathrm{JSNS}^{2}$ neutrino detector

(outer) layers. The scintillation light is detected by $10^{\prime \prime}$ photomultipliers submerged in the liquid scintillator. $\bar{v}_{e}$ is detected by the inverse beta decay (IBD) reaction with free proton in the liquid scintillator,

$$
\bar{v}_{e}+p \rightarrow e^{+}(1 \sim 52 \mathrm{MeV})+n: \quad n+\mathrm{Gd} \rightarrow \mathrm{Gd}^{*} \rightarrow \mathrm{Gd}+\gamma^{\prime} \mathrm{s}(\Sigma E \sim 8 \mathrm{MeV})
$$

The neutron generated in the IBD reaction is absorbed by Gd roughly $30 \mu$ s after the IBD reaction. By taking the coincidence of $e^{+}$signal and the Gd signal, taking into account the delay time of the neutron absorption, $\bar{v}_{e}$ can be identified. This is the same as the case of reactor neutrino detection but the prompt energy is 10 times larger. LSND detector used proton as neutron absorber. Using the Gadolinium, the neutron capture time reduces from $200 \mu \mathrm{s}$ (LSND case) to $30 \mu \mathrm{s}$. Therefore, it is possible to reduce the delayed coincidence window 7 times narrower. In addition, the energy of the $\gamma$-rays emitted after the neutron absorption on proton is only $2.2 \mathrm{MeV}$ for LSND case but JSNS ${ }^{2}$ $(8 \mathrm{MeV})$ is immune to the environmental $\gamma$-ray backgrounds. The light output of the neutrino target scintillator is much more than that of LSND liquid scintillator because LSND group intentionally lowered the scintillation light output to detect Čherenkov light to remove fast neutron background. Due to the high light output, the energy resolution of JSNS $^{2}$ is much better than that of LSND. This 
is an essential feature to see the distortion of the energy spectrum due to the oscillation. As for the statistics, the event rate of JSNS ${ }^{2}$ (phase-I) is 2 times more than LSND one thanks to the stronger beam power and looser event selection criteria, despite its smaller detector mass. Figs. B shows expected energy spectrum of the neutrino and expected sensitivity. JSNS ${ }^{2}$ (the 1st phase) will cover

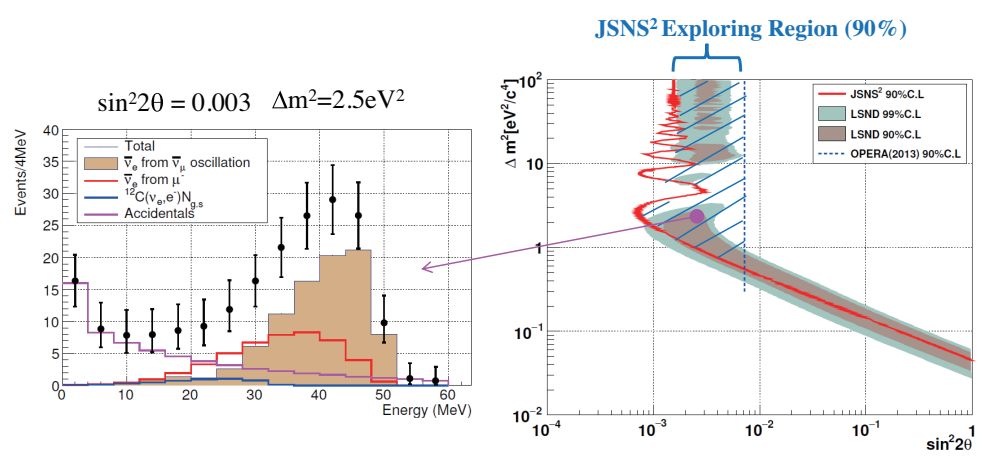

(a)

(b)

Figure 3: (a) Expected energy spectrum. (b) Expected sensitivity.

most of the LSND positive regions. It is also possible to measure the energy spectrum with a good precision.

\section{Status}

At the time of the NuFact conference (2019/August), most of the structural elements were ready. The stainless steel and acrylic tanks were made and waiting for assembly. Fig. $\rightarrow$ shows pictures taken while the drill of the acrylic vessel installation in the stainless steel tank. Both Gd-

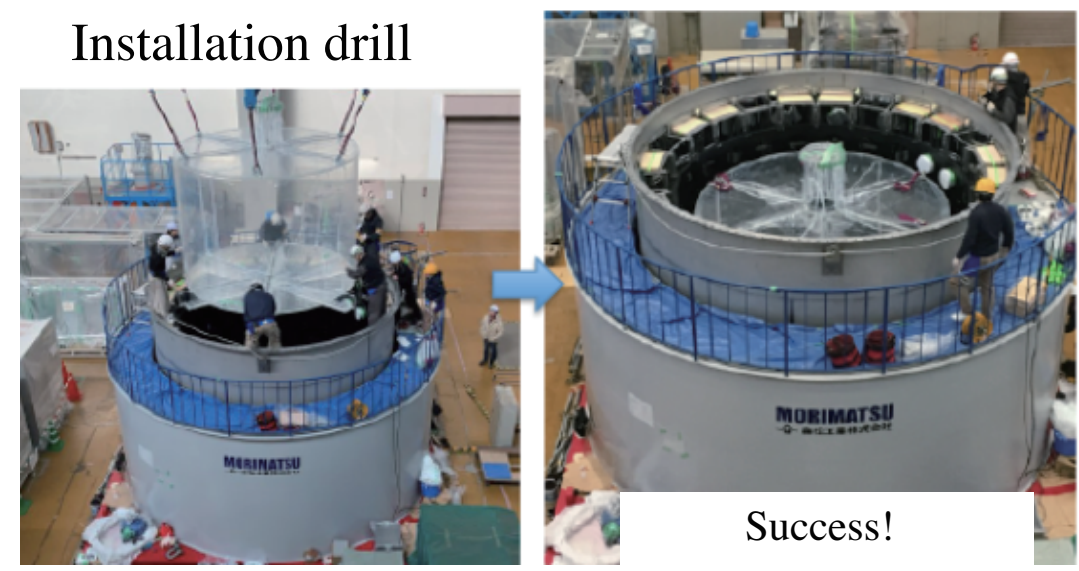

Figure 4: Pictures taken while the drill of the acrylic vessel installation in the stainless steel tank.

loaded and unloaded liquid scintillators are produced and stored in ISO-tanks. PMTs are being calibrated and a part of them are already installed inside the stainless steel tank as shown in Fig. [1. Electronics are also ready and its testing is being performed. Intensive tests of liquid handling 


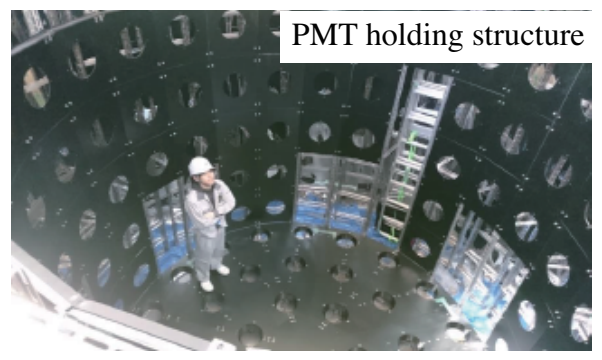

Figure 5: PMT holding structure inside the stainless steel tank.

system are being performed and liquid introduction and extraction procedures are being discussed. Some of those R\&D results have been reported in publications [ 6$]$. With all those activities, we plan to start data taking from early 2020.

\section{Summary}

JSNS $^{2}$ experiment will directly test LSND result using the same neutrino and the same detection method yet much better performances. The 1st phase of JSNS ${ }^{2}$ will start data taking in 2020 and cover the most of the LSND parameter regions. The KDAR experiment is also envisaged to provide unique measurements.

\section{Acknowledgement}

We warmly thank the MLF people, especially, MLF Division leader, the neutron source group, muon group and user facility group for the various kinds of supports. This work is also supported by the JSPS grant-in-aids (Grant Number 16H06344, 16H03967), Japan, and the National Research Foundation of Korea (NRF) Grants 2009-0083526 and 2017K1A3A7A09015973. Special thanks to Daya Bay group for donating the Gd-Loaded liquid scintillator, to Double Chooz group for donating electronics etc., to the RENO group for preparing the liquid scintillator (project number NRF-2017K1A3A7A09016125). Finally, we thank the continuous supports from J-PARC and KEK.

\section{References}

[1] For recent reviews, S. Böser et al., arXiv:1906.01739 (2019) and A. Diaz et al., arXiv:1906.00045 (2019).

[2] LSND collaboration, PRL 77, 3082 (1996), PRL 81, 1774 (1998).

[3] S. Ajimura et al., PTEP 2015(2015) no.6, 063C01.

[4] JSNS ${ }^{2}$ collaboration, arXiv:1310.1437 (2013), arXiv:1705.08629 (2017).

[5] A.A. Aguilar-Arevalo et al., Phys. Rev. Lett. 120, 141802 (2018).

[6] Y. Hino et al., JINST 14 (2019) no.09, T09001, Y. Hino et al., JINST 14 (2019) no.09, P09007, J.S. Park et al., JINST 14 (2019) no.09, T09010. 